\title{
A new proof of a generalization of Gerzon's bound
}

\author{
Gábor Hegedüs ${ }^{1}$
}

Accepted: 1 May 2020 / Published online: 22 November 2020

(c) The Author(s) 2020

\begin{abstract}
In this paper we give a short, new proof of a natural generalization of Gerzon's bound. This bound improves the Delsarte, Goethals and Seidel's upper bound in a special case. Our proof is a simple application of the linear algebra bound method.
\end{abstract}

Keywords Gerzon's bound · Distance problem · Linear algebra bound method

Mathematics Subject Classification 52C45 $\cdot$ 15A03 $\cdot$ 12D99

\section{Introduction}

In this paper we give a linear algebraic proof of the known upper bound for the size of some special spherical $s$-distance sets. This result generalizes Gerzon's general upper bound for the size of equiangular spherical set.

In the sequel, $\mathbb{R}\left[x_{1}, \ldots, x_{n}\right]=\mathbb{R}[\mathbf{x}]$ denotes the ring of polynomials in commuting variables $x_{1}, \ldots, x_{n}$ over $\mathbb{R}$.

Let $\mathcal{G} \subseteq \mathbb{R}^{n}$ be an arbitrary set. Denote by $d(\mathcal{G})$ the set of (non-zero) distances among points of $\mathcal{G}$ :

$$
d(\mathcal{G}):=\left\{d\left(\mathbf{p}_{1}, \mathbf{p}_{2}\right): \mathbf{p}_{1}, \mathbf{p}_{2} \in \mathcal{G}, \mathbf{p}_{1} \neq \mathbf{p}_{2}\right\}
$$

An $s$-distance set is any subset $\mathcal{H} \subseteq \mathbb{R}^{n}$ such that $|d(\mathcal{H})| \leq s$.

Let $(\mathbf{x}, \mathbf{y})$ stand for the standard scalar product. Let $s(\mathcal{G})$ denote the set of scalar products between the distinct points of $\mathcal{G}$ :

$$
s(\mathcal{G}):=\left\{\left(\mathbf{p}_{1}, \mathbf{p}_{2}\right): \mathbf{p}_{1}, \mathbf{p}_{2} \in \mathcal{G}, \mathbf{p}_{1} \neq \mathbf{p}_{2}\right\} .
$$

A spherical $s$-distance set means any subset $\mathcal{G} \subseteq \mathbb{S}^{n-1}$ such that $|s(\mathcal{G})| \leq s$.

Let $n, s \geq 1$ be integers. Define

$$
M(n, s):=\left(\begin{array}{c}
n+s-1 \\
s
\end{array}\right)+\left(\begin{array}{c}
n+s-2 \\
s-1
\end{array}\right) .
$$

$凶$ Gábor Hegedüs

hegedus.gabor@nik.uni-obuda.hu

1 Óbuda University, Bécsi út 96, Budapest 1037, Hungary 
Delsarte, Goethals and Seidel investigated the spherical $s$-distance sets. They proved a general upper bound for the size of a spherical $s$-distance set in [1].

Theorem 1.1 (Delsarte et al. [1]) Suppose that $\mathcal{F} \subseteq \mathbb{S}^{n-1}$ is a set satisfying $|s(\mathcal{F})| \leq s$. Then

$$
|\mathcal{F}| \leq M(n, s) .
$$

Barg and Musin gave an improved upper bound for the size of a spherical $s$-distance set in a special case in [2]. Their proof builds upon Delsarte's ideas (see [1]) and they used Gegenbauer polynomials in their argument.

Theorem 1.2 (Barg and Musin [2]) Let $n \geq 1$ be a positive integer and let $s>0$ be an even integer. Let $\mathcal{S} \subseteq \mathbb{S}^{n-1}$ denote a spherical $s$-distance set with inner products $t_{1}, \ldots, t_{s}$ such that

$$
t_{1}+\cdots+t_{s} \geq 0
$$

Then

$$
|\mathcal{S}| \leq M(n, s-2)+\frac{n+2 s-2}{s}\left(\begin{array}{c}
n+s-1 \\
s-1
\end{array}\right) .
$$

We point out the following special case of Theorem 1.1.

Corollary 1.3 Suppose that $\mathcal{F} \subseteq \mathbb{S}^{n-1}$ is a set satisfying $|s(\mathcal{F})| \leq 2$. Then

$$
|\mathcal{S}| \leq \frac{n(n+3)}{2}
$$

An equiangular spherical set means a two-distance spherical set with scalar products $\alpha$ and $-\alpha$. Let $M(n)$ denote the maximum cardinality of an equiangular spherical set. There is a very extensive literature devoted to the determination of the precise value of $M(n)$ (see $[3,4])$. Gerzon gave the first general upper bound for $M(n) /$

Theorem 1.4 (Gerzon [3, Theorem 8]) Let $n \geq 1$ be a positive integer. Then

$$
M(n) \leq \frac{n(n+1)}{2} \text {. }
$$

Musin proved a stronger version of Gerzon's Theorem in [5, Theorem 1]. He used the linear algebra bound method in his proof.

Theorem 1.5 (Musin [5, Theorem 1]) Let $\mathcal{S}$ be a spherical two-distance set with inner products $a$ and $b$. Suppose that $a+b \geq 0$. Then

$$
|\mathcal{S}| \leq \frac{n(n+1)}{2} .
$$

De Caen gave a lower bound for the size of an equiangular spherical set.

Theorem 1.6 (de Caen [6]) Let $t>0$ be a positive integer. For each $n=3 \cdot 2^{2 t-1}-1$ there exists an equiangular spherical set of $\frac{2}{9}(n+1)^{2}$ vectors.

Our main result is an alternative proof of a natural generalization of Gerzon's bound, which improves the Delsarte, Goethals and Seidel's upper bound in a special case. Our proof uses the linear algebra bound method. The following statement was proved in [7] Theorem 6.1. The original proof builds upon matrix techniques and the addition formula for Jacobi polynomials. 
Theorem 1.7 (Delsarte et al. [7, Theorem 6.1]) Let $s=2 t>0$ be an even number and $n>0$ be a positive integer. Let $\mathcal{S}$ denote a spherical $s$-distance set with inner products $a_{1}, \ldots, a_{t},-a_{1}, \ldots,-a_{t}$. Suppose that $0<a_{i}<1$ for each $i$. Then

$$
|\mathcal{S}| \leq\left(\begin{array}{c}
n+s-1 \\
s
\end{array}\right)
$$

\section{Preliminaries}

We prove our main result using the linear algebra bound method and the Determinant Criterion (see [8, Proposition 2.7]). We recall here this principle for the reader's convenience.

Proposition 2.1 (Determinant Criterion) Let $\mathbb{F}$ be an arbitrary field. Let $f_{i}: \Omega \rightarrow \mathbb{F}$ be functions and $\mathbf{v}_{j} \in \Omega$ elements for each $1 \leq i, j \leq m$ such that the $m \times m$ matrix $B=$ $\left(f_{i}\left(\mathbf{v}_{j}\right)\right)_{i, j=1}^{m}$ is non-singular. Then $f_{1}, \ldots, f_{m}$ are linearly independent.

Consider the set of vectors

$$
\mathcal{M}(n, s):=\left\{\alpha=\left(\alpha_{1}, \ldots, \alpha_{n}\right) \in \mathbb{N}^{n}: \alpha_{1} \leq 1, \sum_{i=1}^{n} \alpha_{i} \text { is even, } \sum_{i=1}^{n} \alpha_{i} \leq s\right\} .
$$

Define the set

$$
\mathcal{N}(n, s)=\left\{\alpha=\left(\alpha_{1}, \ldots, \alpha_{n}\right) \in \mathbb{N}^{n}: \sum_{i=1}^{n} \alpha_{i} \leq s\right\}
$$

Lemma 2.2 Let $n, s \geq 1$ be integers. Then

$$
|\mathcal{N}(n, s)|=\left(\begin{array}{c}
n+s \\
s
\end{array}\right) .
$$

Proof For a simple proof of this fact see [9, Section 9.2 Lemma 4].

We need the following combinatorial statement.

Lemma 2.3 Let $n>0$ be a positive integer and $s>0$ be an even integer. Then

$$
|\mathcal{M}(n, s)|=\left(\begin{array}{c}
n+s-1 \\
s
\end{array}\right) .
$$

Proof It is easy to check that there exists a bijection $f: \mathcal{M}(n, s) \rightarrow \mathcal{N}(n-1, s)$, since $s$ is even. Namely, let $\alpha=\left(\alpha_{1}, \ldots, \alpha_{n}\right) \in \mathcal{M}(n, s)$ be an arbitrary element. Define $f(\alpha):=$ $\left(\alpha_{2}, \ldots, \alpha_{n}\right)$. It is easy to verify that $f(\mathcal{M}(n, s)) \subseteq \mathcal{N}(n-1, s)$ and $f$ is a bijection.

Hence $|\mathcal{M}(n, s)|=|\mathcal{N}(n-1, s)|$ and Lemma 2.2 gives the result.

\section{Proof of Theorem 1.7}

Consider the real polynomial

$$
g\left(x_{1}, \ldots, x_{n}\right)=\left(\sum_{m=1}^{n} x_{m}^{2}\right)-1 \in \mathbb{R}\left[x_{1}, \ldots, x_{n}\right] .
$$


Let $\mathcal{S}=\left\{\mathbf{v}_{1}, \ldots, \mathbf{v}_{r}\right\}$ denote a spherical $s$-distance set with inner products $a_{1}, \ldots, a_{t}$, $-a_{1}, \ldots,-a_{t}$. Here $r=|\mathcal{S}|$. Define the polynomials

$$
P_{i}\left(x_{1}, \ldots, x_{n}\right):=\prod_{m=1}^{t}\left(\left(\left\langle\mathbf{x}, \mathbf{v}_{i}\right\rangle\right)^{2}-\left(a_{m}\right)^{2}\right) \in \mathbb{R}[\mathbf{x}]
$$

for each $1 \leq i \leq r$. Clearly $\operatorname{deg}\left(P_{i}\right) \leq s=2 t$ for each $1 \leq i \leq r$.

Consider the set of vectors

$$
\mathcal{E}(n, s):=\left\{\alpha=\left(\alpha_{1}, \ldots, \alpha_{n}\right) \in \mathbb{N}^{n}: \sum_{i} \alpha_{i} \text { is even, } \sum \alpha_{i} \leq s\right\}
$$

It is easy to verify that if we can expand $P_{i}$ as a linear combination of monomials, then we get

$$
P_{i}\left(x_{1}, \ldots, x_{n}\right)=\sum_{\alpha \in \mathcal{E}(n, s)} c_{\alpha} x^{\alpha},
$$

where $c_{\alpha} \in \mathbb{R}$ are real coefficients for each $\alpha \in \mathcal{E}(n, s)$ and $x^{\alpha}$ denotes the monomial $x_{1}^{\alpha_{1}} \cdot \ldots \cdot x_{n}^{\alpha_{n}}$.

Since $\mathbf{v}_{i} \in \mathbb{S}^{n-1}$, this means that the equation

$$
x_{1}^{2}=1-\sum_{j=2}^{n} x_{j}^{2}
$$

is true for each $\mathbf{v}_{i}$, where $1 \leq i \leq r$. Let $Q_{i}$ denote the polynomial obtained by writing $P_{i}$ as a linear combination of monomials and replacing, repeatedly, each occurrence of $x_{1}^{t}$, where $t \geq 2$, by a linear combination of other monomials, using the relations (3.2).

Since $g\left(\mathbf{v}_{i}\right)=0$ for each $i$, hence $Q_{i}\left(\mathbf{v}_{j}\right)=P_{i}\left(\mathbf{v}_{j}\right)$ for each $1 \leq i \neq j \leq r$.

We prove that the set of polynomials $\left\{Q_{i}: 1 \leq i \leq r\right\}$ is linearly independent. This fact follows from the Determinant Criterion, when we define $\mathbb{F}:=\mathbb{R}, \Omega=\mathbb{S}^{n-1}$ and $f_{i}:=Q_{i}$ for each $i$. It is enough to prove that $Q_{i}\left(\mathbf{v}_{i}\right)=P_{i}\left(\mathbf{v}_{i}\right) \neq 0$ for each $1 \leq i \leq r$ and $Q_{i}\left(\mathbf{v}_{j}\right)=P_{i}\left(\mathbf{v}_{j}\right)=0$ for each $1 \leq i \neq j \leq r$, since then we can apply the Determinant Criterion.

But $P_{i}\left(\mathbf{v}_{i}\right)=\prod_{i=1}^{m}\left(1-a_{m}^{2}\right)$ and $P_{i}\left(\mathbf{v}_{j}\right)=0$, because $\mathcal{S}=\left\{\mathbf{v}_{1}, \ldots, \mathbf{v}_{r}\right\}$ is a spherical $s$-distance set with inner products $a_{1}, \ldots, a_{t},-a_{1}, \ldots,-a_{t}$.

It is easy to check that we can write $Q_{i}$ as a linear combination of monomials in the form

$$
Q_{i}=\sum_{\alpha \in \mathcal{M}(n, s)} d_{\alpha} x^{\alpha}
$$

where $d_{\alpha} \in \mathbb{R}$ are the real coefficients for each $\alpha \in \mathcal{M}(n, s)$. This follows immediately from the expansion (3.1) and from the relation (3.2).

Since the polynomials $\left\{Q_{i}: 1 \leq i \leq r\right\}$ are linearly independent and if we expand $Q_{i}$ as a linear combination of monomials, then all monomials appearing in this linear combination contained in the set of monomials

$$
\left\{x^{\alpha}: \alpha \in \mathcal{M}(n, s)\right\}
$$

for each $i$, hence

$$
r=|\mathcal{S}| \leq|\mathcal{M}(n, s)|=\left(\begin{array}{c}
n+s-1 \\
s
\end{array}\right)
$$

by Lemma 2.3 . 


\section{Concluding remarks}

The following Conjecture is a natural generalization of Theorem 1.5 and a strengthening of Theorem 1.2.

Conjecture 4.1 Let $n \geq 1$ be a positive integer and let $s>0$ be an even integer. Let $\mathcal{S} \subseteq \mathbb{S}^{n-1}$ denote a spherical $s$-distance set with inner products $t_{1}, \ldots, t_{s}$ such that

$$
t_{1}+\ldots+t_{s} \geq 0 \text {. }
$$

Then

$$
|\mathcal{S}| \leq\left(\begin{array}{c}
n+s-1 \\
s
\end{array}\right)
$$

Acknowledgements I am indebted to Lajos Rónyai for his useful remarks.

Funding Open access funding provided by Óbuda University

Open Access This article is licensed under a Creative Commons Attribution 4.0 International License, which permits use, sharing, adaptation, distribution and reproduction in any medium or format, as long as you give appropriate credit to the original author(s) and the source, provide a link to the Creative Commons licence, and indicate if changes were made. The images or other third party material in this article are included in the article's Creative Commons licence, unless indicated otherwise in a credit line to the material. If material is not included in the article's Creative Commons licence and your intended use is not permitted by statutory regulation or exceeds the permitted use, you will need to obtain permission directly from the copyright holder. To view a copy of this licence, visit http://creativecommons.org/licenses/by/4.0/.

\section{References}

1. P. Delsarte, J.M. Goethals, J.J. Seidel, Spherical codes and designs. Geom. Ded. 6(3), 363-388 (1977)

2. A. Barg, O.R. Musin, Bounds on sets with few distances. J. Comb. Theory Ser. A 118(4), 1465-1474 (2011)

3. P.W.H. Lemmens, J.J. Seidel, Equiangular lines. J. Algebra 24, 494-512 (1973)

4. A. Glazyrin, W.H. Yu, Upper bounds for s-distance sets and equiangular lines. Adv. Math. 330, 810-833 (2018)

5. O.R. Musin, Spherical two-distance sets. J. Comb. Theory Ser. A 116(4), 988-995 (2009)

6. D. de Caen, Large equiangular sets of lines in Euclidean space. Electron. J. Combin. 7 Research Paper 55, (2000)

7. P. Delsarte, J. M. Goethals, J. J. Seidel, Bounds for systems of lines, and Jacobi polynomials, in Geometry and Combinatorics (pp. 193-207), (Academic Press, 1991)

8. L. Babai, P. Frankl, Linear algebra methods in combinatorics, (September 1992)

9. D. Cox, J. Little, D. O'Shea, Ideals, Varieties, and Algorithms (Springer, Berlin, 1992)

Publisher's Note Springer Nature remains neutral with regard to jurisdictional claims in published maps and institutional affiliations. 